\title{
Finding optimal route in the Romanian natural gas network
}

\author{
Răzvan RĂDULESCU \\ The Bucharest University of Economic Studies, Bucharest, Romania \\ radulescu.d.razvan@gmail.com \\ Cătălina NEDELCU \\ The Bucharest University of Economic Studies, Bucharest, Romania
}

\begin{abstract}
The Romanian natural gas market is still in its infancy regarding the manners in which the demand and supply match. We are frequently talking about market liquidity or about its dynamic behavior, without considering the monthly losses of the national natural gas system operator that derive from the very lack of matching the supply and demand. The present article proposes a method of overlaying the two in a manner that is feasible for the Romanian natural gas market of 2017, a method that will encapsulate the usage of OBAs (operational balancing agreements) in correlation with a model of allocating the demand and supply as a restricted all-pairs shortest path problem. Based on authors experience, five main variables will be examined: point-of-entry (location and time), volume, time and location of delivery, as well as other sets of data that are particular to this sector. This article will tackle the area of usability of OBAs between suppliers and the degrees of sustainability that such a model offers for clients, therefore the resilience of the system will be analyzed through an optimized transfer of information and accessibility to assets. Due to the complex nature of demand in energy markets and the different requirements set by clients, in which an energy resource can be requested by different sets of users, we will conceive a model that can be applied for natural gas, but also for electric energy production and industrial clients. The scope of this endeavor is to create a tool that will minimize the monthly losses of the national natural gas system operator, as well as its distributors, by allocating, in a fair and unbiased way, the responsibility of fulfilling the requested demand in a certain time unit.
\end{abstract}

Keywords: allocation of demand, sustainability in the natural gas market, adaptable OBA, redistribution of resources, matching of supply and demand.

\section{Introduction}

In order to create a dynamic behavior and liquidity in the natural gas market, the overall construct of the system has to account for the fact that the rights for entry-paid gas must be freely transferred between market participants.

As the volume of gas cannot be accounted for the whole transfer process[A.N: In a streamlined transfer the volume has to go from the producer/importer through the system's transport operator pipelines (SNT-TSO), in the case of Romania SNTGN Transgaz Medias, SA, to a distributor, then a supplier and afterwards to the final customer (it has to have at least three exchange points before it can be utilized)], from importer and/or producer up to its final customer, by a single party [Regulation (EC) No. 715/2009 forbids the system operator to be a vertically integrated company], certain agreements must be in place, which on one hand reserve the capacity for the volume to be transferred or allocated by the TSO (SNT), and on the other hand acquire that volume through a commercial agreement with the producer/importer for a specified volume of gas. 
Therefore, for the implementation of the exit-entry transportation [The entry and exit capacities can be contracted separately by a network user. This means a free allocability of capacities where the gas bought into a system at any entry point is available for delivery (off-take) at any exit point. Thus, any exit point can be supplied from any entry-point without restrictions (European Parliament and the European Council, 2009)] access, a system requires the presence of a VTP (virtual trading point) in order for a shipper to produce/import and use the entry capacity to transport it through a VTP where it can be retraded (sold). This point offers the possibility for the gas to be traded independently of its location and thus to bilaterally transfer titles of gas and/or swap imbalances between network users (DNV KEMA Energy \& Sustainability and COWI Belgium, 2013).

From a financial perspective the more times a respective volume exchanges hands the better balanced is the price for the end user, as well as the fact that the market based balancing generates less residual balancing for the TSO to handle at the end of the day (A.N: The entry-exit system functions with the timeframe/time-unit of one day or less. This permits the network users to optimize and balance their portfolio in correlation with the day-ahead market and within day capacity products (fundamental for short-term trading which in hand facilitates the arbitrage in the market, which increases the liquidity in it). Another effect is that the prices are safeguarded from speculative attempts. From a technical perspective this subsequent trading ought to possess a space where the rights of property are exchanged (VTP) linked with the space where the commercial agreements are done (e.g. BRM and OPCOM, in Romania). The element which is particular to gas markets, in comparison to electricity markets, is that gas is usually commercially contracted through OTCs [Over the counter contracts that settle a bilateral relationship that is often strategic in nature (Duffie, Garleanu, \& Heje Pedersen, 2007, p. 1865)] over longer periods of time, thus resulting in relatively small amounts traded on stock exchanges.

Even though in 2016 over 98\% of gas volumes were exchanged in Romania through OTC (Romanian Ministry of Energy, 2016), the necessity for a functional VPT-TSO-stock exchange triad is necessary in order to create a market driven price for the commodity.

From this perspective the following analysis will propose a method to balance the point-of-entry (location and time), volume, exit-point (location and time), as well as other sets of data that are particular to this sector catered to the requirements of the Romanian natural gas market.

\section{Research methodology}

The aim of this research is to identify the possibility of overlaying the demand and the supply on the Romanian natural gas market, in order to make it a more dynamic and liquid market. Based on the current case study and on the observation of the existing phenomena, authors have developed a method to balance five main variables that will be examined: point-of-entry (location and time), volume, time and location of delivery, as well as other sets of data that are particular to this sector.

In this article, the methods of compensation between market players and the financial aspects of the transactions (e.g. the role of the clearing house, solvability, financial compensation methods and so on) will not be taken into discussion, the sole scope of the method will be the optimized allocation through the variables mentioned above. 
The proposed model for matching the supply and demand will be transposed into a method based on Floyd-Warshall algorithm, that allows us to identify the by comparing all possible paths through the Transmission System Operator (TSO) or National Transport System (SNT) for each pair of vertices (source-customer) in a dense network.

In order to determine the applicability of Floyd-Warshall algorithm, random values were used for the Bucharest-Govora segment of the TSO/SNT. Based on the results, PICBE $\mid 133$ conclusions were drawn.

\section{Concept}

As the presence of a VTP directly impacts the liquidity and behavior of the gas market and therefore is considered beneficial for its operational resilience and balancing of price, its formation will be considered a prerequisite condition (Oxford Institute for Energy Studies, December 2015 ).

Another condition will be the arrangement of sources of supply with similar prices and flow dynamics, as well as a focus on the flexibility/tradability of the volumes of gas that drive prices to their margin.

Having these contextual elements in place their functional relationship has three stages: (I) producers and importers (considered the entry-points of the system) supply the Ante VPT Market (which contract the volumes of natural gas through OTCs and/or acquire them from the stock exchange), (II) the conjunction between property rights (VPT) and allocation (OTS/SNT) permits the already acquired volumes of gas to be delivered freely in the market and (III) the subsequent Post VPT Market that transfers the volumes to the final consumers through distributors and/or to the exit-points through re-trading (see: Figure 1). Stage 3 functions on the basis of bilateral contracts in relation with the end user.

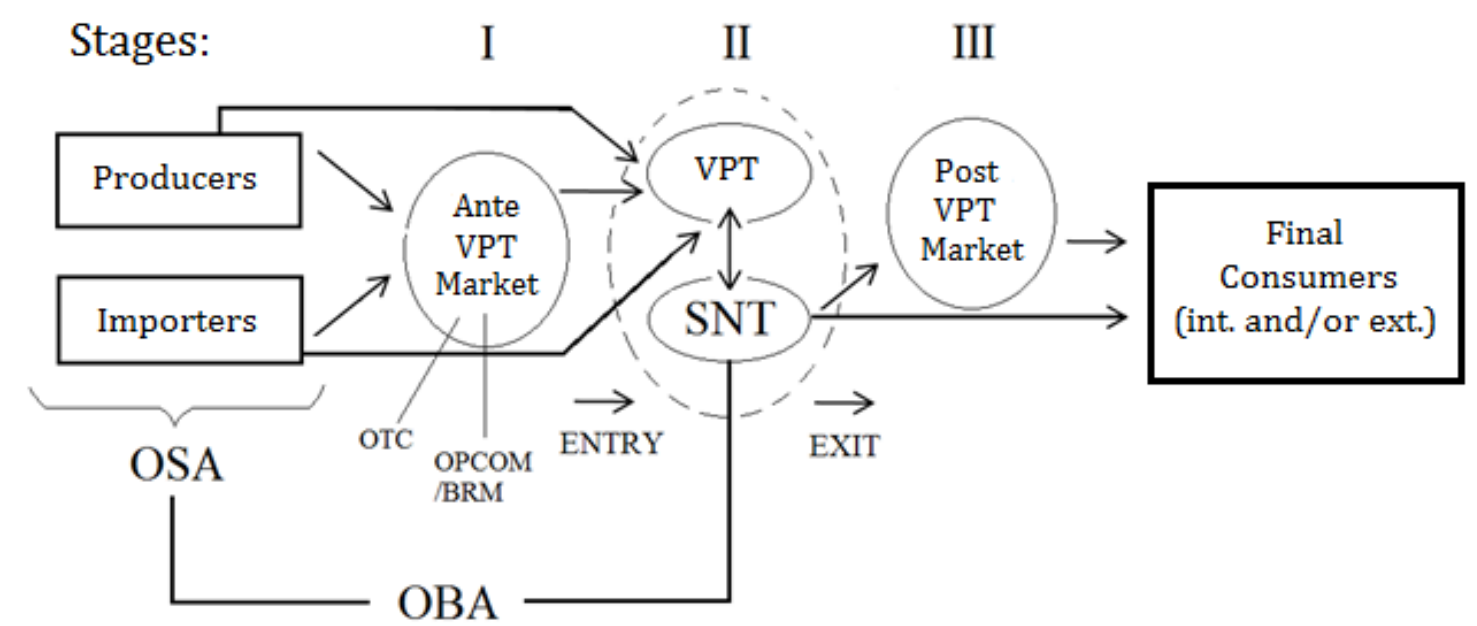

Figure 1. The three distinct stages of matching supply and demand in the gas market

Source: Authors' own research.

The virtual exchange of property (VTP) has to match the physical location of the volumes (STO/SNT data) on the shortest route, in order to optimize the allocation of capacity on the pipelines, and to minimize the resources employed by the TSO to transfer the gas and balance the residual volumes and/or cover them on specific routes. 
Therefore, the proposed model for matching can be transposed into a method based on the shortest path problem with two physical vertices [Singular vertex (or node) is a fundamental unit of graph theory. Two or more unordered vertices form one or more edges; two or more ordered vertices form one or more arcs. Every vertex has a degree (the number of edges incident to it)] (stage I and III) and the process that determines the optimal path (stage II). All of the three stages are time and location dependent, thus the complexity arises from the overlaying of all the possible routes a volume of gas can travel [A.N: Here a main advantage of the PVT is that all possible routes can be compared in a PVT, in comparison to OTCs where the balancing of the network has to be done by the TSO/SNT in a much less dynamic manner (dependent on each supplier and end user's convened structure/timeframe of delivery)] from its entry-point up to its exit-point.

From this moment on, in the working hypothesis, we can refer to stage I and III as vertices and to stage II as their source of variables (the transactions in the VPT give us the quantities, location and time components for the shortest path, all of them are required for the setup of classes inside the method).

As the entry-point and exit-point may be different (e.g. a trader may choose to import a volume one day and the next day to buy them from a local producer, as well as to deliver them to different customers) we can consider these interactions in the market as an all-pairs shortest path problem (Sukumar, Madhumangal, \& Tapan, 2003) in which we have to find the shortest path between two pairs of vertices.

This particular case has all the similarities of optimal routing and can be defined through a Floyd-Warshall algorithm [In certain areas of the TSO/SNT, if the network can be represented as a sparse graph a method for finding the shortest path can be through the Dijkstra algorithm (Dijkstra, 1959)] (Hochbaum, 2014) by comparing all possible paths through the TSO/SNT for each pair of vertices (source-customer) in a dense network. Considering that two classes should be created beforehand (sources and customers) the graph (TSO/SNT network) has to account for all the possible entry and exit points. Afterwards we can consider vertices $V_{s}$ numbered from 1 to $N_{s}$ belonging to the class of sources, and vertices $V_{c}$ numbered from 1 to $\mathrm{N}_{\mathrm{c}}$ belonging to the class of customers.

From this we can consider a function shortestPath $\left(i_{s}, j_{c}, x\right)$ that returns the shortest path possible from $i_{s}$ to $j_{c}$ using the vertices from the set $\{1,2, \ldots, x\}$ as intermediate nodes. From this the scope is to find the shortest way from each $i_{s}$ to each $j_{c}$ using only the point indicated by the TSO/SNT and contained in the set $\{1,2, \ldots, \mathrm{x}+1\}$.

Here the Floyd-Warshall algorithm presents two possibilities:

a) a path that uses vertices in the set $\{1,2, \ldots, x\}$;

b) a path that goes from $i_{s}$ to $x+1$ and then from $x+1$ to $j_{c}$.

The options are compared in themselves and generate the following recursive formula: $\operatorname{shortestPath}\left(i_{s}, j_{c}, x+1\right)=\min \left(\operatorname{shortestPath}\left(i_{s}, j_{c}, x\right)\right.$, $\operatorname{shortestPath}\left(i_{s}, x+1, x\right)+$ $\left.\operatorname{shortestPath}\left(\mathrm{x}+1, \mathrm{j}_{\mathrm{c}}, \mathrm{x}\right)\right)$

This works by computing the shortest path for all $\left(\mathrm{i}_{\mathrm{s}}, \mathrm{j}_{\mathrm{c}}\right)$ pairs for $\mathrm{x}=1$, then $\mathrm{x}=2$ until $\mathrm{x}=\mathrm{N}$ (number of iterations in accordance with the density of the graph), thus at the end the shortest path $\left(i_{s}, j_{c}\right)$ is to be found using the intermediate vertices. 


\section{Results}

In order to examine the applicability of Floyd-Warshall algorithm for any possible point-ofentry and its location $\left(\mathrm{i}_{\mathrm{s}}\right)$, and the subsequent location of a possible point-of exit $\left(\mathrm{j}_{\mathrm{c}}\right)$ we have modeled the Bucharest-Govora segment of the TSO/SNT (SNTGN Transgaz SA Mediaș, 2014, p. 43) using random values for its edges.
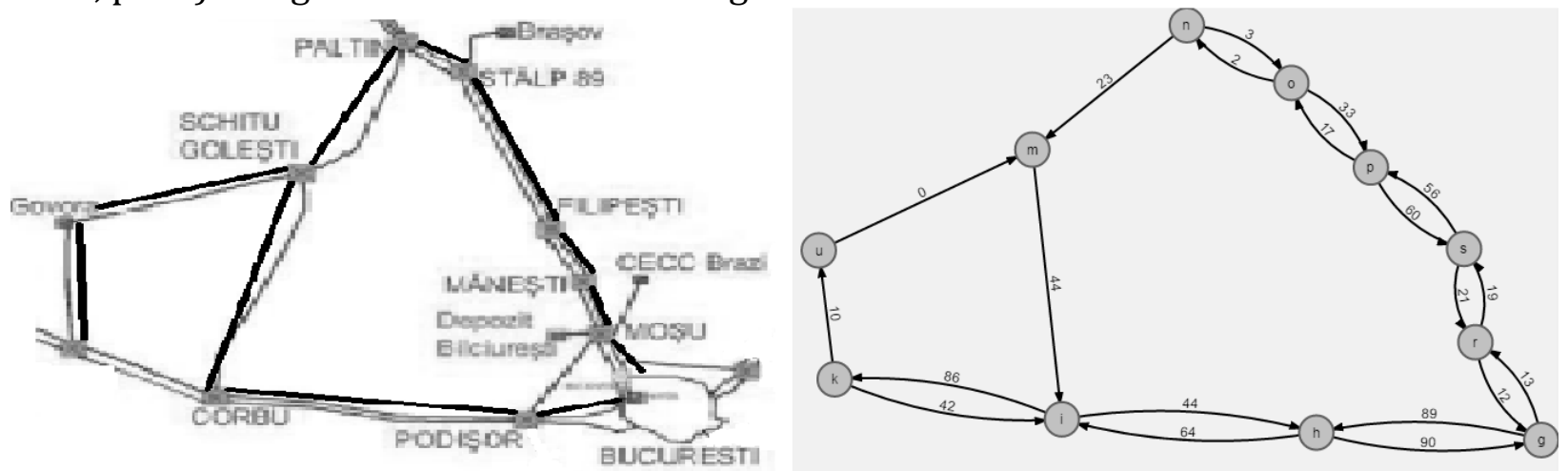

PICBE | 135

Figure 2. Modeling the section of the SNT from Bucharest to Govora as a graph

Source: Authors' own research.

For this simulation the bidirectional flows were unknown, as well as the technical limitations of certain vertices and pipelines (e.g. if they possess compression stations, have different flows or have the capacity to restrict of increase their flow etc.). We note that the simulation was developed using some features of the open-source program: IDP Project of Aleksejs Voroncovs at Chair M9 of Technische Universität München 2015. For this modeled version we have designed bidirectional flows for the pipes linking vertex $\mathrm{p}$ to $\mathrm{k}$ and $\mathrm{p}$ to $\mathrm{n}$. Therefore, we have different distances or time of arrival from one point to the other and vice versa.

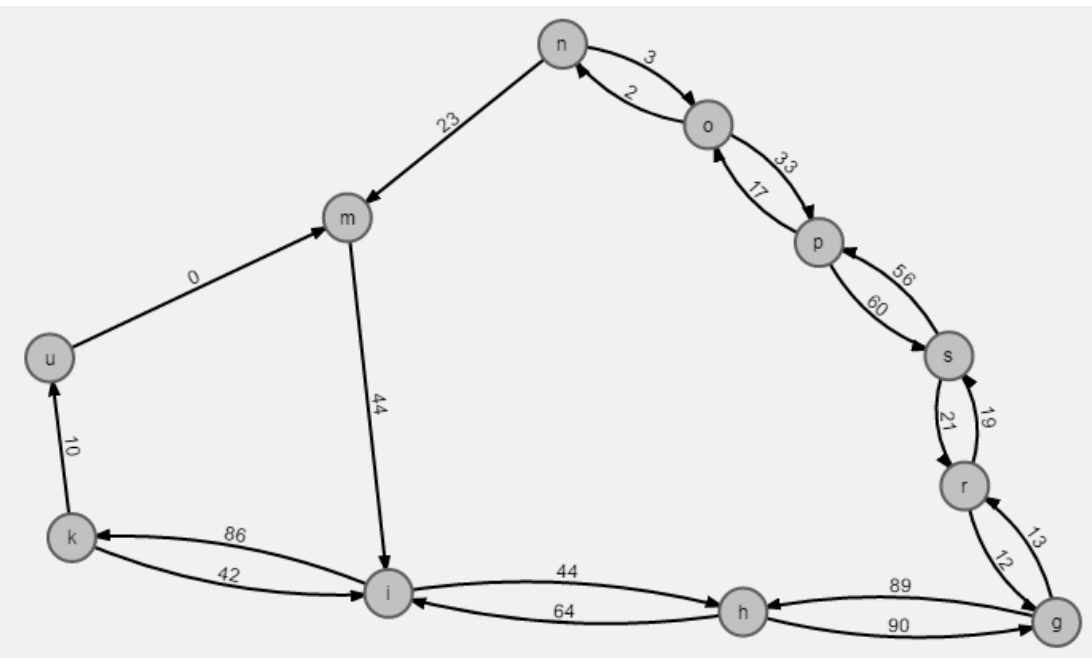

End of the algorithm.

All shortest (or cheapest, respectively) paths have been computed.

The final matrix looks as follows:

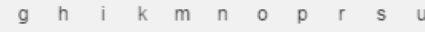

g $\quad 0 \quad 89153239130107 \quad 105 \quad 88 \quad 13 \quad 32 \quad 249$

h $90 \quad 0 \quad 64150160197195178103122160$

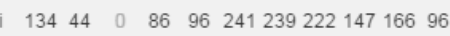

$\begin{array}{llllllllllll}\text { k } 176 \quad 86 & 42 & 0 & 10 & 283 & 281 & 264 & 189 & 208 & 10\end{array}$

m $17888 \quad 44130 \quad 0 \quad 285283266191210140$

$\begin{array}{llllllllllll}\text { n } & 129 & 111 & 67 & 153 & 23 & 0 & 3 & 36 & 117 & 96 & 163\end{array}$

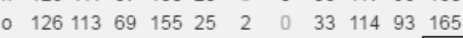

$\begin{array}{llllllllllllll}\text { p } & 93 & 130 & 86 & 172 & 42 & 19 & 17 & 0 & 81 & 60 & 182\end{array}$

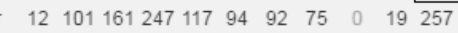

s $\quad \begin{array}{lllllllllll}33 & 122 & 142 & 228 & 98 & 75 & 73 & 56 & 21 & 0 & 238\end{array}$

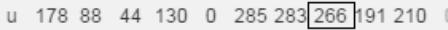

Figure 3. The results of the simulation for every vertex entry-point and exit-point in the graph

Source: Authors' own research. Note: A.N: the distances or time of transport between vertices can be declared zero if inside de model there is a point with certain restrictions (unidirectional flow or restricted flow) and nearby is positioned another point with other characteristics, and the distance between them is negligible (see point $\mathrm{m}$ and $\mathrm{u}$ on the graph in Figure 2.). Floyd-Warshall 
cannot be used for negative distances (edge weights), case which is impossible in the TSO/SNT network, therefore buttressing its usability in this case.

For example the point $\mathrm{p}$ on the graph (equivalent to Filipești) and point $\mathrm{u}$ (equivalent to Govora) have a distance from $\mathrm{p} \rightarrow \mathrm{u}$ of 266 and in comparison the distance from $\mathrm{u} \longrightarrow \mathrm{p}$ is 182 .

We have to mention that the term distance can be substituted to technological time or other relevant unit of measure for accounting the time a certain volume of gas can be transported to a designated vertex (e.g. final customer, distribution network etc.). This method is highly recommended to be used on the TSO/SNT as well as its subsequent distribution networks to evaluate the time and distance a certain volume of gas after the source of gas was secured and before (or even after) the allocation of capacity was realized, this construct can be formalized through operational balancing agreements (OBAs) between market players and the TSO.

\section{Conclusions}

The usage of this method can prove beneficial for the TSO when optimizing the routes of transport for allocated capacities as well as when trying to determine the shortest route (time unit) in which a certain volume of gas can reach a final customer. For this method to operate at its full potential the certain classes of customers have to be determined as well as a detailed mapping of the technical restrictions of the TSO network (for volume and pressure most of all).

This method has also a high degree of applicability under the provisions of EU Regulation 994/2010 where the method and the Floyd-Warshall algorithm can be used also for the transport of naphtha, as an alternative for the use of natural gas, in case of emergency situations as defined in the above regulation (European Parliament and the European Council, 2010).

By liking the distribution systems to the TSO network the cheapest route to the enduser can be determined and allocation scenarios can be developed in respect to the maximum volume that ought to be delivered in a certain unit of time to a batch of customers. By creating another layer which links the distribution-TSO network with the network used for naphtha transports, the time of arrival of alternative fuel stocks can be determined and thus the switch from natural gas to other fuels for thermal power plants can be optimized.

Due to the sensitive nature of the data regarding the Romanian TSO's network and its subsequent distribution networks, to evaluate the proper routes for different volumes of gas and to create action scenarios in case of shortage in the supply of natural gas further batches of information are needed.

From a holistic approach, in which this method would encapsulate also the transport of alternative fuel for thermal and electrical power plants (e.g. naphtha), its transfer routes, beside the TSO network, have to be mapped and integrated in a future model, thus further research is needed.

\section{Bibliography}

Dijkstra, E. (1959). A Note on Two Problems in Connexion with Graphs. Numerische Mathematik, 269-271. 
DNV KEMA Energy \& Sustainability and COWI Belgium. (2013). Study on Entry-Exit Regimes in Gas. Groningen, The Netherlands: European Commission - DG ENERGY.

Duffie, D., Garleanu, N., \& Heje Pedersen, L. (2007). Valuation in Over-the-Counter Markets. The Review of Financial Studies, 1865-1900.

European Parliament and the European Council. (2009). Regulation (EU) No. 715/2009 on conditions for access to the natural gas transmission networks. Bruxelles, Belgium: European Parliament.

European Parliament and the European Council. (2010). Regulation (EU) No. 994/2010 concerning measures to safeguard security of gas supply. Bruxelles, Belgium: European Parliament.

Hochbaum, D. (2014). Lecture Notes for IEOR 266: Graph Algorithms and Network Flows Section 8.9 Floyd-Warshall algorithm for all pairs shortest paths. Department of Industrial Engineering and Operations Research, University of California, Berkeley.

Oxford Institute for Energy Studies. (December 2015 ). The evolution of European traded gas hubs. Oxford, UK: Oxford Institute for Energy Studies.

Romanian Ministry of Energy. (2016, 10 05). Nota de fundamentare OUG 64/2016 pentru modificarea și completarea Legii energiei electrice și a gazelor naturale nr. 123/2012. Retrieved 03 02, 2017, from Guvernul Romaniei: http://gov.ro/ro/guvernul

SNTGN Transgaz SA Mediaș. (2014). Planul de Dezvoltare al Sistemului Național de Transport Gaze Naturale 2014-2023. Mediaș: SNTGN Transgaz SA .

Sukumar, M., Madhumangal, P., \& Tapan, P. K. (2003, March). An Optimal Algorithm to Solve the All-Pairs Shortest Paths Problem on Permutation Graphs. Journal of Mathematical Modelling and Algorithms, 2(1), 57-65. 